\title{
The Square Kilometre Array
}

\section{Peter J Hall ${ }^{1}$}

International SKA Project Office

PO Box 2,7990 AA Dwingeloo, The Netherlands

E-mail: halleskatelescope.org

This short paper provides a description of the international SKA project. It emphasizes key design questions and gives information on current specifications, technology directions and project timescales.

The First MCCT-SKADS Training School

Medicina, Bologna Italy

September $23^{\text {th }}-29^{\text {th }} 2007$

\footnotetext{
$1 \quad$ Speaker
} 


\section{Introduction}

Earlier presentations in this MCCT-SKADS Training School have discussed many contemporary and past radio telescopes, and the wide range of technologies used to realize them. The parabolic dish antenna, with its inherently wide potential frequency range and flexible modes of operation, understandably forms the basis of many stand-alone and interferometer instruments. However, we have also seen that when special-purpose observations are the main drivers, other technology optimizations involving different receptors can be attractive. The SKA design challenge arises not just from the need to make the most sensitive radio telescope ever built, but to do so in a way which preserves flexibility across a range of science applications, including exploration of currently unknown phenomena. This short overview describes the elements of the SKA science case, and outlines key enabling technologies and project developments.

\section{The SKA at a glance}

The SKA is an aperture synthesis radio telescope originally slated to have about $1 \mathrm{~km}^{2}$ of effective collecting area. The plan is that it will be deployed progressively, reaching full sensitivity at frequencies below $10 \mathrm{GHz}$ by 2020 . Higher frequency capability will be added in the decade 2020-30, possibly by means of a different telescope on a site removed from its lower-frequency counterpart.

The motivation for the SKA is to do transformational science which, within the expected $€ 1.5$ billion construction budget, will require the development of a range of new technologies. The level of innovation needed is such that, in real terms, the cost per unit collecting area of the SKA must be at least an order of magnitude lower than that of existing arrays. Characteristics of the SKA include:

- Huge survey and point-source sensitivities;

- High resolutions in the time, frequency and spatial domains; and

- A science case aimed at addressing fundamental physics questions.

There are two short-listed sites for the central part of the instrument: one in the Murchison Shire of Western Australia and the other in the Karoo region of South Africa. A site decision, which will be made largely in the course of government and funding agency negotiations, is expected around 2011.

Over half the SKA collecting area will be contained within a central five kilometre diameter region (Figure 1), with the remaining area distributed in array stations extending to the longest baselines. The science requirements of the SKA drive designers to include transcontinental baselines extending to $3000 \mathrm{~km}$, and Figure 2 shows representative 
configurations centred on the short-listed sites. In present thinking all antennas in the central region will be correlated directly, but on longer baselines station beamforming (signal aggregation) will be done to reduce signal processing and computing requirements. The central array, and indeed portions extending to hundreds of kilometres, will enjoy radio-quiet zone status, with maximum protection afforded close to the centre where interferometer fringe-rate filtering of RFI is least effective.

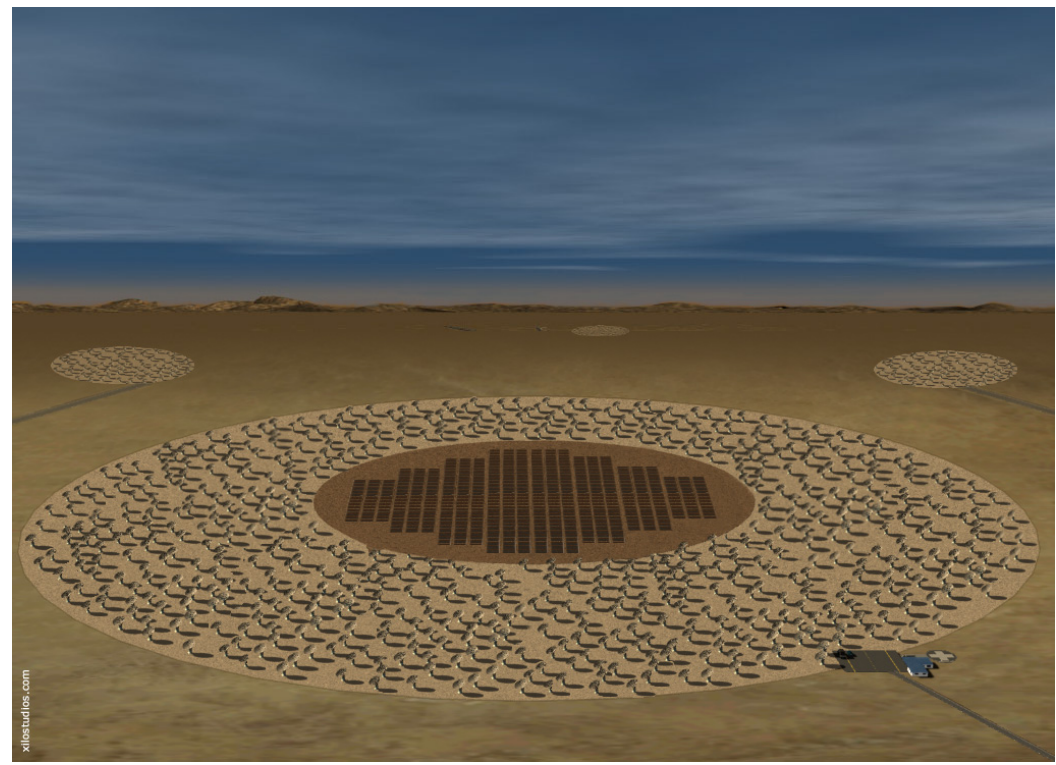

Figure 1: Artist's impression of the SKA core, showing dishes and a mid-band aperture array patch.

With a number of technology options likely to be included in the SKA there is a healthy suite of Pathfinder and Design Study projects underway in various institutes around the world. While this paper draws on material from these projects, it does not provide a complete review. For a comprehensive outline of these exciting advances in astronomy and radio science refer to [1]. 

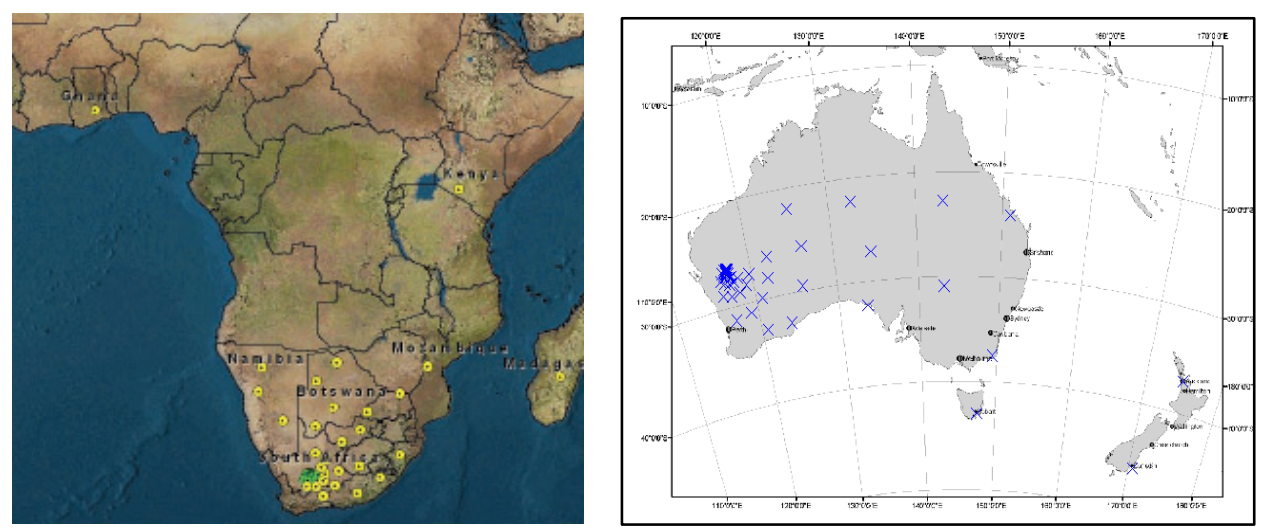

Figure 2: (a) Possible SKA configuration in Southern Africa. Green dots indicate the core in the Karoo region, while yellow dots show stations. (b) Representative Australian SKA configuration. The core is located in the Murchison region of WA, with the blue crosses indicating array stations.

\section{SKA science}

A comprehensive science case for the SKA is available [2] but what is striking is the likely impact of the telescope on basic physics, as well as astronomy. Looking at the "Connecting Quarks with the Cosmos: Eleven Science Questions for the New Century" report [3], one sees that the SKA is set to address over half of the fundamental physics questions listed. Of course, radio astronomy already has a good track record of discovery with four of seven Nobel prizes in astrophysics being awarded to radio astronomers. The SKA, with an ability to see wide fields full of "normal" (as opposed to radio-loud) galaxies, and to fill in the all-important gap between optical views of the Universe and cosmic background fluctuations of the type seen by WMAP, is indeed plausible as a discovery machine of unparalleled capability.

While the SKA science case is large, several areas have been identified as key science projects. These are:

- The first stars and galaxies in the Universe (observing the emergence of structure in the Universe);

- Large scale structure of the Universe (including Dark Energy);

- Origin and evolution of cosmic magnetic fields;

- Gravity in the strong field case via gravitational wave detection ;

- Planet formation, including search for extra-terrestrial intelligence (SETI); and

- Exploration of the unknown. 
More details on each are available in the science case but, for young radio astronomers, it is especially important to devote some time to the last area. It is not just possible but, if history is any guide, quite probable that the best science you will do with the SKA will fall into this category.

\section{Telescope optimization and figures of merit}

Apart from the requirement to maintain flexibility a significant part of the SKA design challenge comes from its wide frequency range: from $70 \mathrm{MHz}$ to $25 \mathrm{GHz}$ in current thinking. A glance at sky and atmospheric noise plots, such as those shown in Figure 3 (below), illustrates the issue. At low frequencies Galactic noise far exceeds the contribution of any modern receiver, while at the highest SKA frequencies water vapour emission from the troposphere starts to be a significant component. Not surprisingly, one may expect different technology performance-cost optimizations across the telescope operating range.

It is useful to think about a couple of basic design parameters which flow from the radiometry relationships discussed in earlier presentations. First of all, we know the telescope flux and antenna temperature fluctuations are related by:

$\Delta \mathrm{S}=2 \mathrm{k} \Delta \mathrm{T}_{\text {ant }} / \mathrm{A}_{\mathrm{eff}}$,

where $A_{\text {eff }}$ is the effective collecting area (i.e., physical area multiplied by factors such as efficiency and projection terms).

In our real radiometer, where the system noise dominates, the output fluctuations can be written as:

$$
\Delta \mathrm{T} \sim \mathrm{T}_{\mathrm{sys}} /(\mathrm{B} \tau)^{1 / 2},
$$

where $T_{\text {sys }}, B$ and $\tau$ are the system equivalent noise temperatures, bandwidth and integration time, respectively. Thus,

$$
\Delta \mathrm{S}=2 \mathrm{k} \mathrm{T}_{\mathrm{sys}} /\left\{(\mathrm{B} \tau)^{1 / 2} \mathrm{~A}_{\mathrm{eff}}\right\}
$$

For small $\Delta \mathrm{S}$, corresponding to easy detection of weak sources, we need a big value of $\mathrm{A}_{\text {eff }} / \mathrm{T}_{\text {sys }}$ and this quantity is often used as a figure-of-merit (FoM) for radio telescopes. It is directly applicable to single-pointing (targetted) observations. When survey observations are made, one can think about a related survey speed FoM which relates to the time taken to cover a particular region of the sky with a given sensitivity. Here we need to invoke the total field-ofview of the telescope; in a typical dish instrument this is related to both the natural FoV and the number of FoVs in a multi-beam system. Using the radiometer equation, and considering the number of pointings needed to survey a given region, one can show that a useful survey speed 
FoM (SSFoM) is $\left(\mathrm{A}_{\text {eff }} / \mathrm{T}_{\text {sys }}\right)^{2}$.FoV . As reference points $\mathrm{A}_{\text {eff }} / \mathrm{T}_{\text {sys }}$ for the VLA at $1.4 \mathrm{GHz}$ is about $210 \mathrm{~m}^{2} \mathrm{~K}^{-1}$, while the SSFoM is about $6000 \mathrm{~m}^{4} \mathrm{~K}^{-2} \mathrm{deg}^{2}$.

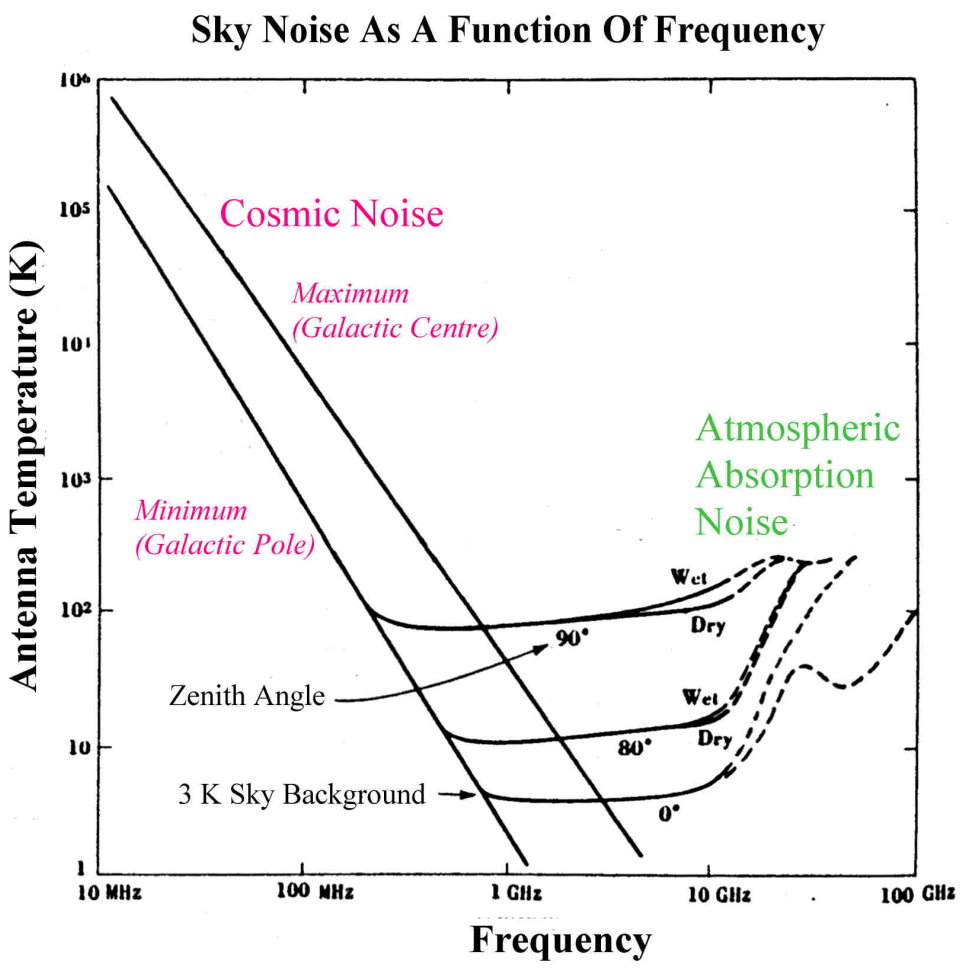

Figure 3: Plot of antenna temperature as a function of frequency. In radio engineering terms the received noise power in a given bandwidth is directly proportional to the antenna temperature. Adapted from [4].

A great deal of time in the SKA project has gone into thinking about what is the maximum affordable, and minimum scientifically useful, $\mathrm{A}_{\mathrm{eff}} / \mathrm{T}_{\text {sys }}$ in various frequency bands. Furthermore, away from the extreme upper and lower SKA frequencies in Figure 3, careful thought is needed about the most cost-effective way of reaching a desired $\mathrm{A}_{\mathrm{eff}} / \mathrm{T}_{\text {sys }}$ quotient. Is it better to build more collecting area, or spend money on lower-noise receivers? Of course, at the low frequencies where Galaxy emission dominates completely the system temperature, area is the only solution and technologies like sparse aperture arrays - which give effective areas scaling with wavelength squared - are very attractive. Conversely, at higher SKA frequencies, where the sky temperature is lower, careful control over system temperature pays dividends. In this regime reducing receiver noise by cryo-cooling, and limiting the view by the receptor of the "hot" ground via secondary optics arrangements, are both conveniently done within dish antenna designs. 
Considering the range of SKA science to be done, it is also necessary to think about the trade-offs in reaching a given survey speed. With the SSFoM defined as above, is it better to strive for a high $\mathrm{A}_{\text {eff }} / \mathrm{T}_{\text {sys }}$ or should we look to obtain the same survey performance by increasing the FoV? Of course, more $A_{\text {eff }} / T_{\text {sys }}$ is always best since it also increases the point-source sensitivity but the reality is that, with modern technology, generating a wide FoV can be more attractive economically than building more collecting area or cooling receivers. Recent thinking in the SKA project has given pre-eminence to the survey speed specification but has also provided limiting values of $A_{\text {eff }} / T_{\text {sys }}$ which engineers must deliver. For reference, these draft top-level specifications are set out in Table 1 (below). Specifications are given for "low-risk" technology implementations and (in parentheses) for more ambitious technologies, implementation of the latter being contingent upon demonstration by regional Pathfinders and Design Studies. A much more complete discussion of the SKA specifications, and the science and technology bases for them, is available in [5].

TABLE 1. Draft SKA Specifications as at November, 2007

\begin{tabular}{|c|c|c|c|}
\hline Parameter & $\begin{array}{l}\text { Phase } 1 \\
10 \% \text { SKA }\end{array}$ & $\begin{array}{c}\text { Phase } 2 \\
\begin{array}{c}\text { Full SKA at low \& mid } \\
\text { bands }\end{array}\end{array}$ & $\begin{array}{c}\text { Phase } 3 \\
\text { Full SKA }\end{array}$ \\
\hline $\begin{array}{r}\text { Low }(\mathrm{GHz}) \\
\operatorname{High}(\mathrm{GHz})\end{array}$ & $\begin{array}{l}0.200 \\
3\end{array}$ & $\begin{array}{l}0.070 \\
3(8)\end{array}$ & $\begin{array}{c}0.070 \\
25(35)\end{array}$ \\
\hline \begin{tabular}{rr|} 
Survey speed $\left(\mathrm{m}^{4} \mathrm{~K}^{-2} \mathrm{deg}^{2}\right)^{*}$ & \\
$70-200 \mathrm{MHz}$ \\
$200-500 \mathrm{MHz}$ \\
$0.7 \mathrm{GHz}$ \\
$1.4 \mathrm{GHz}$ \\
$3 \mathrm{GHz}$ \\
$8 \mathrm{GHz}$ \\
$25 \mathrm{GHz}$
\end{tabular} & $\begin{array}{ll}- & \\
1.2 \times 10^{6} & \left(1 \times 10^{7}\right) \\
3 \times 10^{5} & \left(1 \times 10^{7}\right) \\
- & \end{array}$ & $\begin{array}{ll}3 \times 10^{9} & \left(2 \times 10^{10}\right) \\
1.3 \times 10^{7} & \left(2 \times 10^{10}\right) \\
1.2 \times 10^{8} & \left(2 \times 10^{10}\right) \\
6 \times 10^{7} & \left(6 \times 10^{8}\right) \\
2.6 \times 10^{6} & \left(1.4 \times 10^{7}\right) \\
4 \times 10^{5} & \left(2 \times 10^{6}\right)\end{array}$ & $\begin{array}{ll}3 \times 10^{9} & \left(2 \times 10^{10}\right) \\
1.3 \times 10^{7} & \left(2 \times 10^{10}\right) \\
2.4 \times 10^{8} & \left(2 \times 10^{10}\right) \\
1 \times 10^{9} & \left(6 \times 10^{9}\right) \\
1.4 \times 10^{7} & \\
8 \times 10^{6} & \end{array}$ \\
\hline $\begin{array}{r}\text { Min. sensitivity at } 45^{\circ}\left(\mathrm{A}_{\text {eff }} / T_{\text {sys }}\right)\left(\mathrm{m}^{2} \mathrm{~K}^{-1}\right)^{*} \\
70-200 \mathrm{MHz} \\
200-500 \mathrm{MHz} \\
0.5-3 \mathrm{GHz} \\
8 \mathrm{GHz} \\
25 \mathrm{GHz} \\
\end{array}$ & $\begin{array}{l}700 \\
250\end{array}$ & $\begin{array}{l}4000(10000) \\
10000 \\
4500(10000) \\
2500(5000)\end{array}$ & $\begin{array}{l}4000(10000) \\
10000 \\
10000 \\
10000 \\
5000\end{array}$ \\
\hline $\begin{array}{rr}\text { Configuration } & \text { core }(<1 \mathrm{~km}) \\
\text { inner }(<5 \mathrm{~km}) \\
\text { mid }(<180 \mathrm{~km}) \\
\text { outer (up to at least } 3000 \mathrm{~km})\end{array}$ & $\begin{array}{l}5 \% \\
7.5 \% \\
10 \% \\
10 \%\end{array}$ & $\begin{array}{r}20 \% \\
50 \% \\
75 \% \\
100 \%\end{array}$ & $\begin{array}{r}20 \% \\
50 \% \\
75 \% \\
100 \% \\
\end{array}$ \\
\hline 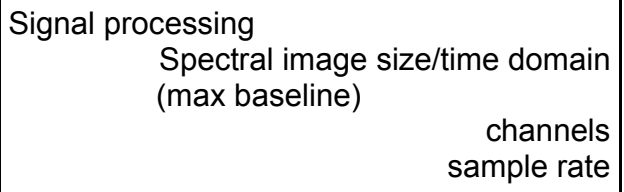 & $\begin{array}{l}5 \mathrm{~km} \\
16384 \\
0.1 \mathrm{~ms}\end{array}$ & $\begin{array}{l}10 \mathrm{~km} \\
16384(32768) \\
0.1 \mathrm{~ms}\end{array}$ & $\begin{array}{l}20(50) \\
16384(32768) \\
0.1 \mathrm{~ms}\end{array}$ \\
\hline
\end{tabular}


For those familiar with earlier SKA goals, notice that the project has moved some way from the primary specification of $A_{\text {eff }} / T_{\text {sys }}=20000 \mathrm{~m}^{2} \mathrm{~K}^{-1}$. (It was this goal, coupled with a canonical system temperature of $50 \mathrm{~K}$, which led to an $\mathrm{A}_{\text {eff }}$ of $10^{6} \mathrm{~m}^{2}$ or one square kilometre).

Of course, obtaining and dealing with a wide FoV can be quite challenge: one needs antennas capable of seeing a large part of the sky and, just as importantly, an ICT machine comprising data transport links and information processing stages with sufficient capacity to handle the information generated by the wide-field system. Figure 4 is a diagram showing a view of the SKA that is a little different to commonly seen ones. It emphasizes the ICT nature of the telescope, as well as conveying that the wide range of operating frequencies makes it necessary to use a number of antenna types.

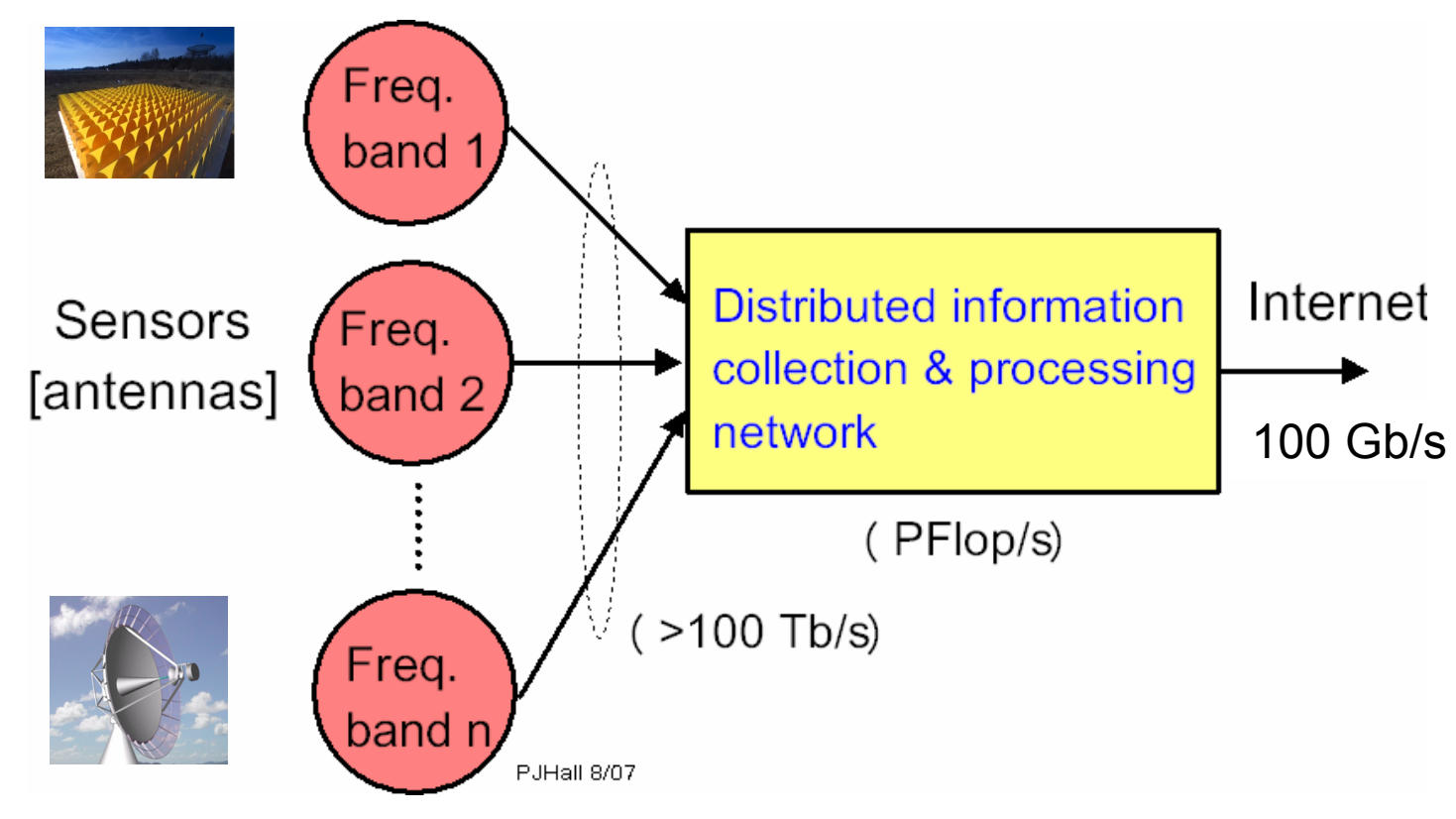

Figure 4: View of the SKA as an ICT machine, with a series of optimized sensors providing inputs to the distribution network and processing engine.

Part of the multi-dimensional optimization facing SKA designers is to decide what fraction of the component budget to spend on e.g. antennas, versus that to be spent on electronics. Fortunately, some guiding analyses are available [6] and new tools [7] make examination of "what if" scenarios increasingly easier.

SKA will be the successor to a range of new radio telescopes (including LOFAR) which are increasingly ICT based. In general these machines: 
- Exploit the convergence of radio and ICT engineering;

- Open new observing parameter space via more flexibility, hopefully enabling discovery;

- Achieve gains via functionality/cost improvements (based partially on consumer electronics and computing technology), and include capabilities such as collecting area re-use ("multi-fielding") at lower frequencies;

- Pose new optimization challenges in which new players can be as effective as old ones in key areas; and

- Can be built in stages, mitigating engineering risk and offering progressive science capability.

Undoubtedly, SKA and its Pathfinders (LOFAR, ATA etc) are incubators for much new astronomy and technology because of the radio - ICT marriage.

\section{SKA Reference Design technologies}

Prior to 2006 the SKA spent several years of assessing the prospects of various antenna technology options [8]. A number of "Reference Design" technologies have now been chosen [9]; at the highest and lowest frequencies the choice of parabolic dishes and aperture arrays (AAs), respectively, is clear (see previous section). In the SKA context, the electronicallybeamformed AA below $300 \mathrm{MHz}$ is sparse, which means that the mutual coupling between dipole-like elements is not strong (the element separation is certainly $>\lambda / 2$ ); this is also the situation in LOFAR and similar arrays. Figure 5a shows some LOFAR antennas designed to operate in the range $110-240 \mathrm{MHz}$.

At frequencies above about $1.4 \mathrm{GHz}$, where $\sim 15 \mathrm{~m}$ dishes are used as optical beamformers, variants of conventional "single-pixel" feeds are used. The twist in the SKA application is that the feeds are expected to be very wide-band types, most likely 10:1 in bandwidth ratio. One such feed has been demonstrated by the Allan Telescope Array (ATA) and is shown in Figure 5b.

While the technology solutions in the SKA low and high bands are clear enough, the optimum mid-band ( $300 \mathrm{MHz}-1.5 \mathrm{GHz})$ technology is yet to be established. In the Reference Design an interesting hybrid technology is proposed. This implementation uses first-level beamforming done optically with a dish but also invokes a phased array at the dish's focus. The "phased array feed" (PAF) uses a dense focal-plane receptor array, and digital beamforming technology, to give perhaps 30 beams from the dish, each one having an area on the sky commensurate with the natural (single-pixel) field-of-view of the antenna. Ultimately the fieldof-view expansion is limited by the off-axis aberration in the focal-plane and the resultant complexity in forming off-axis beams. Nevertheless, attainable field-of-view expansions of 30 or so are highly attractive in reaching the required SKA survey specifications. Figure $5 \mathrm{c}$ is an 
artist's impression of a PAF on a $12 \mathrm{~m}$ dish; note that several hundred close-packed elemental feed antennas are used to form the 30 or so beams from the dish. The PAF technology is being demonstrated in the ASKAP and APERTIF projects, in Australia and The Netherlands, respectively.

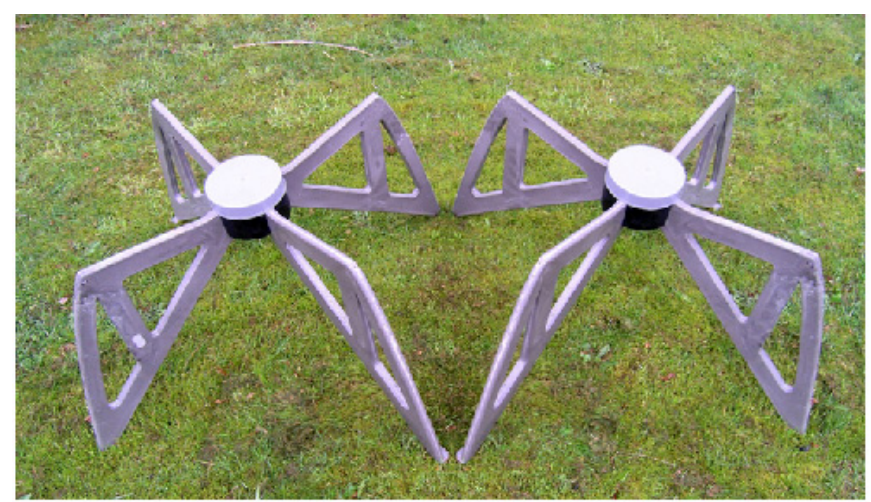

(a)

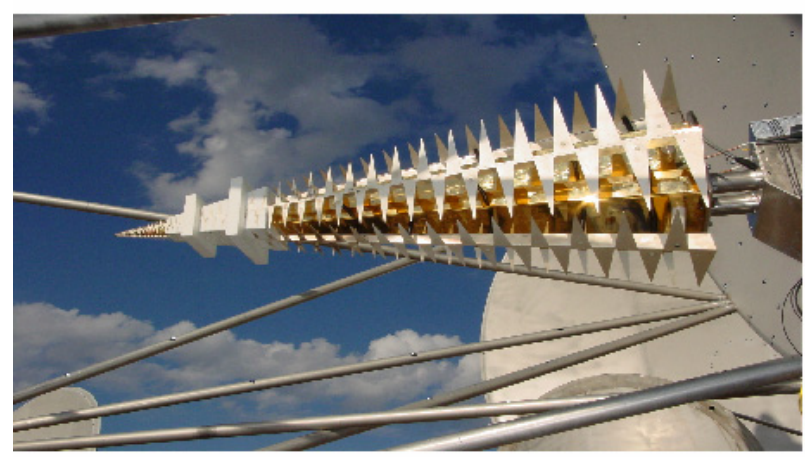

(b)

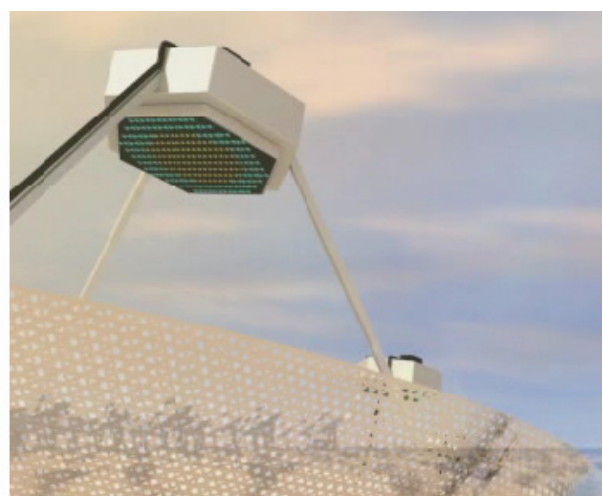

(c)

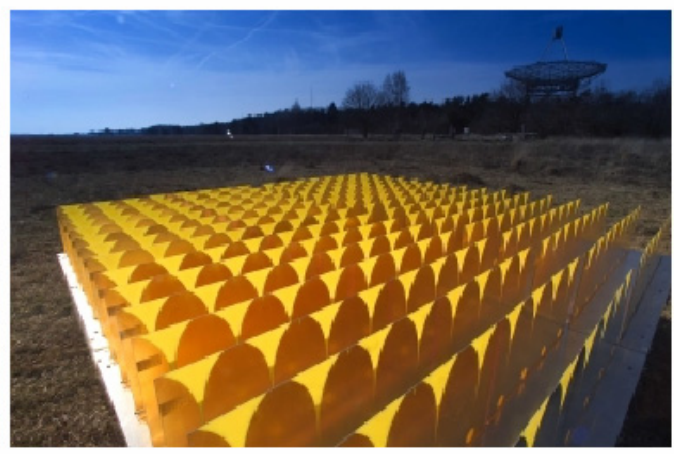

(d)

Figure 5: SKA Reference Design components. (a) LOFAR high-band dipole antennas similar to those which may be used as low-band SKA antennas. (b) A broadband Allen Telescope Array feed, which forms a starting point for decade-bandwidth high-band SKA feeds. (c) Artist's impression of a mid-band dish plus phased array feed (PAF) solution. (d) Mid-band, dense, aperture phased array tile prototype produced by ASTRON.

A second mid-band technology being developed in Europe involves dense aperture arrays. As in the PAF, the elements in these arrays are highly coupled electrically. In this case though the dish reflector has been entirely discarded and the whole collecting area is composed of dense AAs; fields-of-view are formed electronically by either analogue or digital first-stage processing and there may be many later-stage beams generated within a given FoV. As in the sparse AAs, the first-stage beamforming operation can be repeated many times over, giving both wide and independent fields-of-view. The dense array uses a number of elemental feeds per wavelength of operation and the close-packing gives the array some dish-like properties. For example, the effective area remains more or less constant with frequency (compared with 
the $\lambda^{2}$ dependence for the sparse array) and the beams produced should have quite low (and hopefully easily calibrated) sidelobes. Dense AAs are being demonstrated by the European EMBRACE and 2-PAD projects within the FP6 SKADS program [10]. A dense AA tile, based on Vivaldi end-fire antennas, is shown in Figure 5d.

Roughly speaking, a given field-of-view requires the same number of receivers, whether one uses single-pixel feeds, PAFs, AAs, or any other receptor techology [6]. Wide FoVs therefore require many receivers - several million in the case of an AA in which the full FoV potential is reached. Cheap, high performance receivers, are thus an enabling technology for the SKA. In one approach, illustrated in Figure 6, a consumer-grade RF CMOS integrated-circuit process has been used to make a fully-integrated mid-band SKA receiver.

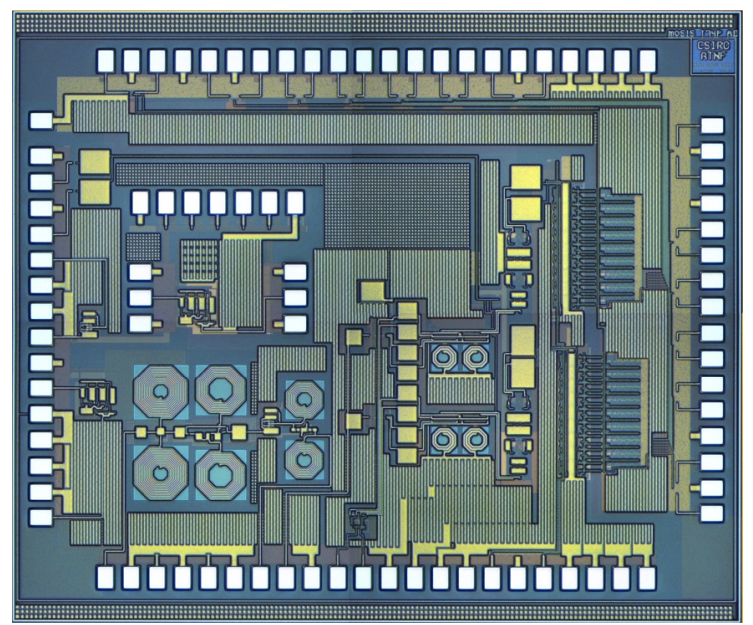

Figure 6: Photograph of a prototype mid-band SKA integrated receiver based on low-cost CMOS technology. RF signals in the range $0.7-1.8 \mathrm{GHz}$ enter at the left, with in-phase and quadrature data streams (at up to 512 mega-samples per second) leaving at the right. The chip is about $3.5 \mathrm{~mm}$ on a side. Courtesy Suzy Jackson, CSIRO.

It is not yet clear which is the best technology for mid-band SKA although the great versatility of the AA makes it highly likely that it will be included in some form. It is uniquely suitable for all-sky monitoring (e.g. in transient searches) and could be included in this role even if the PAF solution proves more economical for deployment over large areas. Of course, one also has to consider that neither the PAF nor AA wide field-of-view options may be able to be implemented at attractive performance and cost points, leading SKA designers to keep in mind a solution involving sparse aperture arrays - perhaps operating at frequencies somewhat higher than LOFAR - and dishes equipped with single-pixel feeds. Possible technology combinations based on various development outcomes are canvassed more fully in [5] but here it is sufficient to note that the SKA Preparatory Phase study commencing in 2008 will produce designs which incorporate demonstrated results from the various international SKA Pathfinder and Design Studies. 
Antennas are of course the most visible part of the SKA but it is important to bear in mind other substantial engineering challenges. For example, the following areas will certainly figure large in the minds of the team producing the Telescope design:

- High speed data transport

- $\mathrm{Tb} / \mathrm{s}$ of data flow from each station, out to scales of hundreds of kilometres

- $100 \mathrm{~Gb} / \mathrm{s}$ trans-continental and trans-oceanic links are needed

- The longest links involve telecommunications companies and national research networks

- Signal processing

- Peta-operations per second will be required in the correlator and time-domain digital signal processors

- Highly scaleable architectures will be essential

- Post-processing and information management

- New super-computer architectures will be needed to cope with real-time SKA processing needs

- Archive and sharing of data will be a major challenge

- Large-scale software engineering

$\circ$ Estimates of 1000 person-years of effort have been made, even for constrained complexity in SKA operational modes

- Infrastructure

- Civil, electrical, communications projects will be very challenging at SKA sites, all of which will be remote regardless of which nation hosts the instrument

- Operations and support

- Scheduling, maintenance and other areas of array operation will be far more complex than for existing instruments.

The general SKA development approach is one of frequent astronomy and engineering iteration to refine specifications and to promote design convergence as rapidly as possible. There is a strong emphasis on technology demonstration, effectively retiring risks as early as possible. This demonstration is being done in the context of regional Pathfinders and Design Studies, amounting to a direct investment of more than $€ 150 \mathrm{M}$ (much more if SKA-related ventures such as LOFAR, FAST etc are counted). These programs focus on aggressive cost reduction strategies, as well promotion of the industry engagement which will be needed to deliver SKA on required timescales.

\section{The SKA project}

From its beginnings in the early 1990s the SKA has been a global collaboration and, in recent times, some 18 countries are represented. The governing policy body is the International SKA Steering Committee (ISSC) and, since 2003, an International SKA Project Office (ISPO) 
has had executive and coordination roles in advancing the project. The ISPO relies on a number of working groups, several of which have associated specialist task forces. This is an important feature of the SKA organization because, apart from four individuals in the ISPO, all other contributors to the project are located in various regional institutes. The project is entering a new era in 2008 with the commencement of a European-sponsored FP7 program known as the SKA Preparatory Phase Study, or PrepSKA. PrepSKA contains seven work packages and is designed to take the SKA to the point where it can be funded. For reference, the various activities are:

- WP1 - PrepSKA management

- WP2 - SKA system design

- WP3 - Continuing site selection process

- WP4 - Governance

- WP5 - Industry and procurement policy

- WP6 - Funding model

- WP7 - Implementation strategy

Most of the work packages run for three years. The exception is WP2, which is a fouryear package designed to produce a top-level SKA system design (including costing) by 2012. A major function of WP2 is to establish an ISPO Central Design Integration Team (CDIT), consisting of about 15 engineers located at the ISPO host institute, the Jodrell Bank Centre for Astrophysics in the UK. The demonstration of central and regional interactions is important since this will undoubtedly feature in any SKA construction model. Most importantly, representatives of various funding agencies will be involved with SKA personnel in framing options for governance, financial and associated policy models.

Figure 7 shows a timeline for the SKA project from which the concurrency of the PrepSKA timescale with those of regional Pathfinder and Design Studies is obvious. With this overlap in mind, PrepSKA is designed to coordinate, augment and interface with the large-scale efforts occurring in regional groups. In financial terms, PrepSKA is a €25M program comprising both EU and international SKA components. 


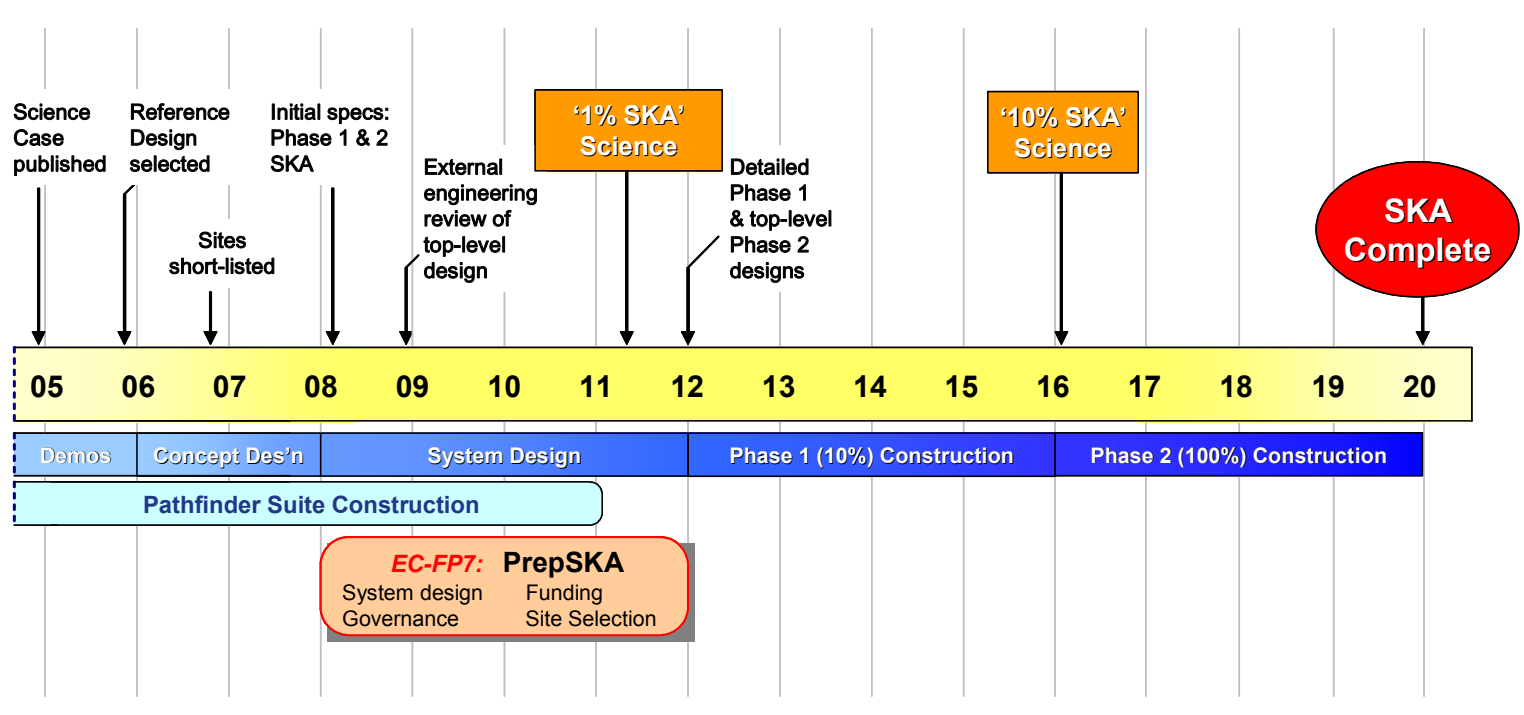

Figure 7: Timeline for the SKA project. Note the overlapping Pathfinder and PrepSKA timescales.

\section{Conclusion}

The SKA project is now established as an international mega-science endeavour and many more details, both technical and organizational, are available at www.skatelescope.org. With the pace of the project advancing rapidly, frequent checks of the site - especially the memo series - is recommended.

\section{References}

[1] ISPO, Status of pathfinder telescopes and design studies, available at http://www.skatelescope.org/PDF/Annex_3 of_Draft_SKA_10Sep07.pdf.

[2] C. Carilli and S. Rawlings (Eds), Science with the SKA, New Astronomy Reviews, $\underline{\mathbf{4 8}}$, Elsevier, 2004.

[3] National Academies (Board on Physics and Astronomy), Connecting quarks with the cosmos, National Academies Press, 2003.

[4] J. Kraus, Radio Astronomy, McGraw-Hill, 1966.

[5] R. Schilizzi et al., Draft specifications for the SKA, available at http://www.skatelescope.org/PDF/Draft_specifications 10Sep07.pdf

[6] J. Bregman, System optimization of multi-beam aperture synthesis arrays for survey performance, in The SKA: An Engineering Perspective, P. Hall (Ed), Springer, Dordrecht, 2005. 
[7] A. Chippendale et al., SKAcost - a tool for SKA cost and performance estimation, SKA Memo 92, 2007, available at http://www.skatelescope.org/PDF/memos/memo_92.pdf

[8] P. Hall, The SKA - an international engineering perspective, in The SKA: An Engineering Perspective, P. Hall (Ed), Springer, Dordrecht, 2005.

[9] ISPO, Reference Design for the SKA, SKA Memo 69, 2006, available at http://www.skatelescope.org/PDF/memos/69 ISPO.pdf

[10] SKADS Consortium, Radio astronomy enters a new era, 2007, available at http://www.skadseu.org/PDF/SKADS_brochure.pdf 